\title{
BDD vs. Constraint-Based Model Checking: An Experimental Evaluation for Asynchronous Concurrent Systems ${ }^{\star}$
}

\author{
Tevfik Bultan \\ Department of Computer Science, University of California \\ Santa Barbara, CA 93106, USA \\ bultan@cs.ucsb.edu
}

\begin{abstract}
BDD-based symbolic model checking has been successful in verification of a wide range of systems. Recently, constraint-based approaches, which use arithmetic constraints as a symbolic representation, have been used in symbolic model checking of infinite-state systems. We argue that use of constraint-based model checking is not limited to infinite-state systems. It can also be used as an alternative to BDDbased model checking for systems with integer variables which have finite but large domains. In this paper we investigate the trade-offs between these two approaches experimentally. We compare the performance of BDD-based model checker SMV to the performance of our constraintbased model checker on verification of several asynchronous concurrent systems. The results indicate that constraint-based model checking is a viable option for verification of asynchronous concurrent systems with large integer domains.
\end{abstract}

\section{Introduction}

Model checking has been used in verification of diverse applications ranging from hardware protocols [McM93] to software specifications [ $\mathrm{CAB}^{+}$98]. The success of model checking has been partially due to use of efficient data structures like Binary Decision Diagrams (BDDs) which can encode boolean functions in a highly compact format [Bry86]. The main idea in BDD-based symbolic model checking is to represent sets of system states and transitions as boolean logic formulas, and manipulate them efficiently using the BDD data structure $\left[\mathrm{BCM}^{+}\right.$90].

An important property of the BDD data structure is that it supports operations such as intersection, union, complement, equivalence checking and existential quantifier elimination (used to implement relational image computations) which also happen to be the main operations required for model checking. However, an efficient encoding for boolean domains may not be efficient for all variable types. For example, BDD-based model checkers can be very inefficient in representing arithmetic constraints $\left[\mathrm{CAB}^{+}\right.$98].

\footnotetext{
* This work was supported in part by NSF grant CCR-9970976 and a University of California Regents' Junior Faculty Fellowship.
} 
Another shortcoming of the BDD representation is its inability to encode infinite domains. Without abstraction, BDDs cannot be used for analyzing infinitestate systems - even those with just one unbounded integer. BDDs encode all underlying datatypes as boolean variables; hence all BDD-based model checkers inherently require the underlying types to be bounded.

Recently, arithmetic constraints have been used as a symbolic representation in model checking [AHH96,BGP97]. For example, HyTech, a symbolic model checker for hybrid systems, encodes real domains using linear constraints on real variables [AHH96]. We developed a model checker for integer based systems which uses Presburger arithmetic (integer arithmetic without multiplication) constraints as its underlying state representation [BGP97,BGP99]. Our model checker uses the Omega library $\left[\mathrm{KMP}^{+} 95\right]$ to manipulate Presburger arithmetic constraints. In [DP99] model checking queries are converted into constraint logic programs, and a CLP $(\mathrm{R})$ library is used to verify concurrent systems by mapping integer variables to real domains.

Constraint representations allow verification of infinite-state systems since they can represent variables with infinite domains. There are algorithms for intersection, union, complement, equivalence checking and existential quantifier elimination for both real and integer constraint representations mentioned above. However model checking becomes undecidable for infinite-state systems. Hence the fixpoint computations are not guaranteed to converge. This problem is addressed using conservative approximation techniques [BGP99] which guarantee convergence but do not guarantee a definite answer, i.e., the model checker 1) may report that the property is verified, 2) provide a counter-example demonstrating violation of the property, or 3) report that the analysis is inconclusive.

Using arithmetic constraints one can also represent variables with finite domains. We just have to add additional constraints which show the range of values that an integer variable can take. An interesting issue is, then, comparing the performance of BDD-based model checking to constraint-based model checking for finite-state systems with integer variables.

In this paper we compare the performance of a BDD-based model checker (SMV [McM93]) and a constraint-based model checker (our model checker based on Omega library [BGP97,BGP99]) in verification of asynchronous concurrent systems with integer variables. On the extreme case where integer variables can take only two values, they can be treated as boolean variables and represented using BDDs. Using a constraint-representation would be very inefficient in such a case. On the other hand, although BDD-based model checkers are not capable of handling systems with unbounded integers, if the variables are restricted to a finite set of values, they can be represented using a set of boolean variables using a binary encoding. Our goal in this paper is to investigate the middle ground between these two extremes where the integer variables are neither unbounded nor have only two possible valuations.

We perceive efforts in constraint-based model checking as not only a way to solve infinite-state verification problems, but also as a way to deal with problems with large variable domains using formalisms that are more expressive than 
boolean logic formulas. However, because of the added expressive power, manipulation algorithms for these formalisms have higher complexity than corresponding algorithms for BDDs. These powerful algorithms may not be worthwhile to use for small domains because of their high complexity. On the other hand, for large domains their complexity maybe justified. The question is, when is the use of integer constraint representations justified instead of BDD encodings? In this paper we investigate this issue experimentally on verification of asynchronous concurrent systems.

The rest of the paper is organized as follows. We first discuss other related approaches to symbolic model checking in Sect. 2. In Sect. 3, we give a brief overview of symbolic model checking. After presenting the example concurrent systems in Sect. 4, we discuss the experimental results we obtained using BDD and constraint-based model checkers in Sect. 5. Finally, we present our conclusions and future directions.

\section{Related Work}

Another approach to infinite-state model checking is to use automata-based representations. Automata can be used to represent arithmetic constraints on unbounded integer variables [WB95,BKR96,KSA98]. An arithmetic constraint on $k$ integer variables is represented by a $k$-track automata that accepts a string if it corresponds to a $k$-dimensional integer vector (in binary representation) that satisfies the corresponding arithmetic constraint. Again, since the automata representation supports the necessary operations, it can be used in symbolic model checking.

The constraint and automata-based representations provide two different ways of implementing model checking computations for systems with unbounded integer variables. In [SKR98] these two approaches are compared experimentally for reachability analysis of several concurrent systems. The results show no clear winner. On some problem instances the constraint representation is superior, on some others automata representation is. In automata-based representations, restricting variables to fixed finite domains ends up converting the automata representation to a model isomorphic to BDDs [KSA98]. Hence, for the experiments we conduct in this paper the automata-based representation is equivalent to BDD-based model checking.

Using a BDD-based model checker such as SMV [McM93] for checking systems with integer variables can easily result in inefficient encodings of arithmetic constraints $\left[\mathrm{CAB}^{+}\right.$98]. It is pointed out both in [YMW97] and [CAB ${ }^{+}$98] that SMV can be very inefficient in constructing BDDs for integer variables. This inefficiency can be resolved for linear arithmetic constraints by using a better variable ordering as explained in $\left[\mathrm{CAB}^{+} 98\right]$. For non-linear constraints, however, there is no efficient BDD representation [Bry86]. In [CABN97] SMV is augmented with a constraint solver for non-linear constraints. This technique is not applicable to the systems we analyze in this paper because of the restrictions put on the types of systems that can be analyzed. 
Another approach to dealing with integer variables in BDD-based model checking is to use abstractions. In [CGL92], a conservative abstraction method is presented for model-checking infinite-state programs. The main idea is to produce a finite model of the program using a suitable abstraction technique (e.g., congruence modulo an integer, single-bit abstraction, symbolic abstraction), and then to check the property of interest on the abstraction. For systems such as the ones we analyze in this paper, finding a good abstraction maybe as difficult as proving the invariants of the system. On the other hand, automated abstractions such as the ones presented in $\left[\mathrm{HKL}^{+} 98\right]$ are not strong enough to eliminate the integer variables in the systems we analyze in this paper.

\section{$3 \quad$ Symbolic Model Checking}

In model checking, the system to be analyzed is represented as a transition system $T S=(S, I, R)$ with a set of states $S$, a set of initial states $I \subseteq S$, and a transition relation $R \subseteq S \times S$. The transition system model is never explicitly generated in symbolic model checking. For example, BDD-based model checkers represent transition relation $R$ as a set of boolean logic formulas.

A popular temporal logic for specifying temporal properties of transition systems is Computation Tree Logic (CTL) [CES86] which consists of a set of temporal operators (the next-state operators EX and AX, the until operators EU and AU, the invariant operators EG and AG, and the eventuality operators $\mathrm{EF}$ and $\mathrm{AF}$ ) for specifying temporal properties.

Our goal in model checking a system $T S=(S, I, R)$ and a temporal property $p$ is (we use $p$ to denote its truth set) : 1) either to prove that the system $T S$ satisfies the property $p$ by showing that $I \subseteq p$, or 2) to demonstrate a bug by finding a state $s \in I \cap \neg p$, and generating a counter-example path starting from $s$.

Assume that there exists a representation for sets of states which supports tests for equivalence and membership. Then, if we can represent the truth set of the temporal property $p$, and the set of initial states $I$ using this representation, we can check the two conditions listed above. If the state space is finite, explicit state enumeration would be one such representation. Note that as the state space of a concurrent system grows, explicit state enumeration will become more expensive since the size of this representation is linearly related to the number of states in the set it represents. Unfortunately, state space of a concurrent system increases exponentially with the number of variables and concurrent components. This state space explosion problem makes a simple implementation of the explicit state enumeration infeasible.

Another approach is to use a symbolic representation for encoding sets of states. For example, a logic formula which is semantically interpreted as a set of states, can be used as a symbolic representation. Boolean logic formulas (stored using the BDD data structure) are the most common symbolic representation used in model checking $\left[\mathrm{BCM}^{+}\right.$90]. Recently, we used Presburger 
arithmetic (integer arithmetic without multiplication) formulas for the same purpose [BGP97,BGP99].

Model checking procedures use state space exploration to compute the set of states which satisfy a temporal property. Fixpoints corresponding to truth sets of temporal formulas can be computed by iteratively aggregating states using pre-condition computations (which correspond to the next state operator EX). Temporal properties which require more than one fixpoint computation can be computed recursively starting from the inner fixpoints and propagating the partial results to the outer fixpoints.

All temporal properties in CTL can be expressed using boolean connectives, next state operator EX, and least fixpoints. For example, $\mathrm{EF} p \equiv \mu x \cdot p \vee \operatorname{EX} x$. The least fixpoint of a monotonic functional can be computed by starting from the bottom element (i.e., false $\equiv \emptyset$ ) and by iteratively applying the functional until a fixpoint is reached.

Assume that Symbolic is the data type used for encoding sets of states. In order to implement a symbolic model checker based on Symbolic data type we need the following procedures:

Symbolic Not(Symbolic): Given an argument that represents a set $p \subseteq S$, it returns a representation for $S-p$.

Symbolic And(Symbolic,Symbolic): Given two arguments representing two sets $p, q \subseteq S$, it returns a representation for $p \cap q$.

Symbolic Or(Symbolic,Symbolic): Given two arguments representing two sets $p, q \subseteq S$, it returns a representation for $p \cup q$.

Symbolic EX(Symbolic): Given an argument that represents a set $p \subseteq S$, it returns a representation for the set $\left\{s \mid \exists s^{\prime} . s^{\prime} \in p \wedge\left(s, s^{\prime}\right) \in R\right\}$.

Boolean Equivalent(Symbolic, Symbolic) : Given two arguments representing two sets $p, q \subseteq S$, it returns true if $p \equiv q$, returns false otherwise.

Using the procedures described above, given a temporal formula, we can compute its truth set by computing the fixpoint that corresponds to that temporal formula.

The computation of the procedure EX involves computing a relational image. Given a set $p \subseteq S$ and a relation $X \subseteq S \times S$ we use $X p$ to denote relational image of $p$ under $X$, i.e., $X p$ is defined as restricting the domain of $X$ to set $p$, and returning the range of the result. Note that we can think of relation $X$ as a functional $X: 2^{S} \rightarrow 2^{S}$. Then, $X p$ denotes the application of the functional $X$ to set $p$.

Let $R^{-1}$ denote the inverse of the transition relation $R$. Then $\operatorname{EX} p \equiv R^{-1} p$, i.e., functional EX corresponds to the inverse of the transition relation $R$. Hence, we can compute the procedure EX using a relational image computation. Most model checkers represent transition relation $R$ in a partitioned form to make the relational image computation more efficient [BCL91].

Any representation which is able to encode the set of initial states $I$ and the set of atomic properties $A P$, and supports the above functionality can be used as a symbolic representation in a model checker. We call such a representation 
an adequate language for model checking [KMM $\left.{ }^{+} 97\right]$. For example, for finitestate systems, boolean logic would be one such representation. It is possible to implement procedures for negation, conjunction, disjunction and equivalence checking of boolean logic formulas. If we can represent the transition relation $R$ as a boolean logic formula, then relational image computation $R^{-1} p$ can be computed by conjuncting the formula representing $R^{-1}$ and the formula representing $p$, and then eliminating the variables in the domain of the resulting relation using existential quantifier elimination. BDDs are an efficient data structure for representing boolean logic formulas, and they support all the functionality mentioned above [Bry86]. They have been successfully used for model checking $\left[\mathrm{BCM}^{+}\right.$90,McM93]. However, they can not encode infinite variable domains.

Recently, we developed a model checker for systems with unbounded integer variables using Presburger arithmetic formulas as a symbolic representation [BGP97]. There are effective procedures for manipulating Presburger formulas which support the above functionality - for example Omega Library implements a set of such procedures $\left[\mathrm{KMP}^{+}\right.$95]. We implemented a model checker using Omega Library as our symbolic manipulator. However, model checking computations become undecidable for infinite domains, i.e., the fixpoint computations corresponding to temporal properties may not always converge for infinite domains. We addressed this issue in [BGP99] using conservative approximations.

\section{Example Concurrent Systems}

The examples we use in this paper have the following characteristics: 1) they are all asynchronous concurrent systems, and 2) they all use shared integer variables to control their synchronization. We think this type of systems are especially suitable for constraint-based representations. Most of our examples are from [And91].

We represent each concurrent system with a set of events, where each event is considered atomic (Fig. 1). The state of a program is determined by the values of its data and control variables. If a variable $v$ is used in an event, then the symbol $v^{\prime}$ denotes the new value of $v$ after the action is taken. If $v^{\prime}$ is not mentioned in an event, then we assume that its value is not altered by that event. Each event specification defines a transition relation over the Cartesian product of the domains of the variables in the system. The transition relation of the overall concurrent system is defined as the union of the transition relations of all events in the system.

Bakery algorithm, shown in Fig. 1 for two processes, is a mutual exclusion algorithm. The algorithm we present above is the coarse grain solution [And91] which can be further refined to implement without fetch-and-add instructions.

In Fig. 1 we show a solution to sleeping barber problem [And91]. The barber allows a new customer into the shop with the event $e_{\text {next }}$. The customer gets a chair by calling the event $e_{\text {haircut }_{1}}$ (as long as their is an available chair). Then the barber starts the haircut with event $e_{n e x t_{2}}$. When the haircut is finished the 
Program: Bakery

Data Variables: $a, b$ : positive integer

Control Variables: $p c_{1}:\left\{T_{1}, W_{1}, C_{1}\right\}, p c_{2}:\left\{T_{2}, W_{2}, C_{2}\right\}$

Initial Condition: $a=b=0 \wedge p c_{1}=T_{1} \wedge p c_{2}=T_{2}$

Events:

$e_{T_{1}}: p c_{1}=T_{1} \wedge p c_{1}^{\prime}=W_{1} \wedge a^{\prime}=b+1$

$e_{W_{1}}: p c_{1}=W_{1} \wedge(a<b \vee b=0) \wedge p c_{1}^{\prime}=C_{1}$

$e_{C_{1}}: p c_{1}=C_{1} \wedge p c_{1}^{\prime}=T_{1} \wedge a^{\prime}=0$

$e_{T_{2}}: p c_{2}=T_{2} \wedge p c_{2}^{\prime}=W_{2} \wedge b^{\prime}=a+1$

$e_{W_{2}}: p c_{2}=W_{2} \wedge(b<a \vee a=0) \wedge p c_{2}^{\prime}=C_{2}$

$e_{C_{2}}: p c_{2}=C_{2} \wedge p c_{2}^{\prime}=T_{2} \wedge b^{\prime}=0$

Program: Barber

Data Variables: cinchair, cleave, bavail, bbusy, bdone: positive integer

Control Variables: $p c_{1}:\{1,2\}, p c_{2}:\{1,2\} p c_{3}:\{1,2\}$

Initial Condition: cinchair $=$ cleave $=$ bavail $=$ bbusy $=$ bdone $=0$

$\wedge p c_{1}=p c_{2}=p c_{3}=1$

Events:

$e_{\text {haircut }_{1}}: p c_{1}=1 \wedge p c_{1}^{\prime}=2 \wedge$ cinchair $<$ bavail $\wedge$ cinchair $^{\prime}=$ cinchair +1

$e_{\text {haircut }_{2}}: p c_{1}=2 \wedge p c_{1}^{\prime}=1 \wedge$ cleave $<$ bdone $\wedge$ cleave $^{\prime}=$ cleave +1

$e_{\text {next }_{1}} \quad: p c_{2}=1 \wedge p c_{2}^{\prime}=2 \wedge$ bavail $^{\prime}=$ bavail +1

$e_{\text {next }_{2}} \quad: p c_{2}=2 \wedge p c_{2}^{\prime}=1 \wedge$ bbusy $<$ cinchair $\wedge$ bbusy ${ }^{\prime}=$ bbusy +1

$e_{\text {finish }_{1}}: p c_{3}=1 \wedge p c_{3}^{\prime}=2 \wedge$ bdone $<$ bbusy $\wedge$ bdone $e^{\prime}=$ bdone +1

$e_{\text {finish }_{2}}: p c_{3}=2 \wedge p c_{3}^{\prime}=1 \wedge$ bdone $=$ cleave

Program: Readers-Writers

Data Variables: $n r, n w$ : positive integer

Initial Condition: $n r=n w=0$

\section{Events:}

$e_{\text {reader-enter }}: n w=0 \wedge n r^{\prime}=n r+1$

$e_{\text {reader-exit }}: n r>0 \wedge n r^{\prime}=n r-1$

$e_{\text {writer-enter }}: n r=0 \wedge n w=0 \wedge n w^{\prime}=n w+1$

$e_{\text {writer-exit }}: n w>0 \wedge n w^{\prime}=n w-1$

Program: Bounded-Buffer

Parameterized Constant: size : positive integer

Data Variables: available, produced, consumed: positive integer

Initial Condition: produced $=$ consumed $=0 \wedge$ available $=$ size

Events:

$e_{\text {produce }}: 0<$ available $\wedge$ produced ${ }^{\prime}=$ produced $+1 \wedge$ available $^{\prime}=$ available -1

$e_{\text {consume }}$ : available $<$ size $\wedge$ consumed ${ }^{\prime}=$ consumed +1

$\wedge_{\text {available }}^{\prime}=$ available +1

Program: Circular-Queue

Parameterized Constant: size : positive integer

Data Variables: occupied, head, tail, produced, consumed: positive integer

Initial Condition: occupied $=$ head $=$ tail $=$ produced $=$ consumed $=0$

Events:

$e_{\text {produce }}:$ occupied $<$ size $\wedge$ occupied ${ }^{\prime}=$ occupied $+1 \wedge$ produced $^{\prime}=$ produced +1

$\wedge\left(\right.$ tail $=$ size $\wedge$ tail $^{\prime}=0 \vee$ tail $<$ size $\wedge$ tail $^{\prime}=$ tail +1$)$

$e_{\text {consume }}:$ occupied $>0 \wedge$ occupied $^{\prime}=$ occupied $-1 \wedge$ consumed $^{\prime}=$ consumed +1

$\wedge($ head $=$ size $\wedge$ head $=0 \vee$ head $<$ size $\wedge$ head $=$ head +1$)$

Fig. 1. Example concurrent systems used in the experiments 
barber executes $e_{\text {done }_{1}}$, and waits $\left(e_{\text {done }_{2}}\right)$ till the customer leaves by executing the event $e_{\text {haircut }_{2}}$.

A well-known algorithm for readers-writers problem is also presented in Fig. 1. The invariant of the readers-writers problem states that at any time there would be either no writers accessing the database or no readers, and the number of writers should never be more than one.

Two algorithms given in Fig. 1 present bounded-buffer implementations. Both these systems have a parameterized constant size which specifies the size of the buffer. Since size is parameterized the systems given above should be correct for any value of size.

In Table 1 we list the invariants the systems presented above have to satisfy.

Table 1. List of problem instances used in the experiments

\begin{tabular}{|c|c|}
\hline Problem Instance & Property \\
\hline BAKERY & $\mathrm{AG}\left(\neg\left(p c_{1}=C_{1} \wedge p c_{2}=C_{2}\right)\right)$ \\
\hline BARBER & $\begin{array}{l}\mathrm{AG}(\text { cinchair } \geq \text { cleave } \wedge \text { bavail } \geq \text { bbusy } \geq \text { bdone } \\
\wedge \text { cinchair } \leq \text { bavail } \wedge \text { bbusy } \leq \text { cinchair } \wedge \text { cleave } \leq \text { bdone })\end{array}$ \\
\hline BARBER-1 & $\mathrm{AG}($ cinchair $\geq$ cleave $\wedge$ bavail $\geq$ bbusy $\geq$ bdone $)$ \\
\hline BARBER-2 & $\mathrm{AG}($ cinchair $\leq$ bavail $\wedge$ bbusy $\leq$ cinchair $)$ \\
\hline BARBER-3 & $\mathrm{AG}($ cleave $\leq$ bdone $)$ \\
\hline READERS-WRITERS & $\mathrm{AG}((n r=0 \vee n w=0) \wedge n w \leq 1)$ \\
\hline BOUNDED-BUFFER & $\begin{array}{l}\mathrm{AG}(\text { produced }- \text { consumed }=\text { size }- \text { available } \\
\wedge 0 \leq \text { available } \leq \text { size })\end{array}$ \\
\hline BOUNDED-BUFFER-1 & $\mathrm{AG}($ produced - consumed $=$ size - available $)$ \\
\hline BOUNDED-BUFFER-2 & $\mathrm{AG}(0 \leq$ available $\leq$ size $)$ \\
\hline BOUNDED-BUFFER-3 & $\mathrm{AG}(0 \leq$ produced - consumed $\leq$ size $)$ \\
\hline CIRCULAR-QUEUE & $\begin{array}{r}\mathrm{AG}(0 \leq \text { produced }- \text { consumed } \leq \text { size } \\
\wedge \text { produced }- \text { consumed }=\text { occupied })\end{array}$ \\
\hline CIRCULAR-QUEUE-1 & $\mathrm{AG}(0 \leq$ produced - consumed $\leq$ size $)$ \\
\hline CIRCULAR-QUEUE-2 & $\mathrm{AG}($ produced - consumed $=$ occupied $)$ \\
\hline
\end{tabular}

\section{Experimental Evaluation}

We translated the examples given in Fig. 1 to the SMV input language. For each concurrent process we used the process declaration in SMV which supports asynchronous composition. SMV converts all integer variables to a binary representation since it is a BDD-based model checker. We used an uninitialized variable that always preserves its value to represent the parameterized constant size in the bounded-buffer and circular-queue systems.

Our omega library based model checker accepts a Presburger arithmetic formula for each event in the input system. It forms the global transition relation by combining these formulas disjunctively. It uses asynchronous composition to combine two concurrent components. It is not efficient to map variables with small domains (such as program counters) to integer variables. So, for the exam- 
ples with control variables we used control point partitioning to eliminate the control variables [BGP99].

To compare the performances of SMV and OMC (Omega library Model Checker) we assigned a finite domain to each integer variable. We generated 16 different instances for each concurrent system by restricting the integer variables to different ranges. We started with a range of $0 \leq i<2^{3}$ for each integer variable $i$ (which makes it possible to represent each variable $i$ with 3 boolean variables in SMV) and increased it until $0 \leq i<2^{26}$ (which requires 26 boolean variables for each integer variable).

In Figs. 2 and 3 we show the performances of both SMV and OMC in terms of execution time and memory usage. We ran all our experiments on an Intel Pentium III PC (500MHz, 128 MByte main memory) running Solaris. Each graph shows experiments on one concurrent system. Data points in each individual graph is generated by only changing the range of values that integer variables are allowed to take. The $x$ axis in these graphs show the number of boolean variables required for the binary encoding of each integer variable (which ranged from 3 to 26 in our experiments). So, for each point in the graph, the range of each integer variable $i$ in the concurrent system verified in that particular experiment is $0 \leq i<2^{x}$.

In our initial experiments we observed that the execution time and the memory usage of SMV increases exponentially with the number of boolean variables required for the binary encoding of each integer variable (which corresponds to a linear increase in the size of the domains of the integer variables). This exponential increase can be observed in Figs. 2 and 3.

The worst-case complexity of the BDD representation is exponential in the number of boolean variables it represents. The exponential increase in execution time and memory usage of SMV is a realization of this worst-case complexity. However, as observed by Chan et al. [CAB+98] and Yang et al. [YMW97] this is because of the inefficient representation of integer variables in SMV and can be improved using a better variable ordering.

BDD representation is very sensitive to variable ordering [Bry86]. In SMV, given two integer variables $i$ and $j$, all the boolean variables representing variable $i$ either precede all the boolean variables representing $j$ or vice versa. With such an ordering the BDD representing a constraint such as $i=j$ has exponential size in the number of boolean variables. However, if the order of boolean variables representing $i$ and $j$ are interleaved the same constraint has a linear BDD representation $\left[\mathrm{CAB}^{+}\right.$98]. William Chan developed macros which generate such an interleaved order for SMV. Using his macros, we tested the SMV system again for the examples given in Fig. 1. As seen in Figs. 2 and 3, with this variable ordering the execution time and the memory usage of SMV increases linearly with the number of boolean variables required for the binary encoding of each integer variable (which corresponds to a logarithmic increase in the size of the domains of the integer variables).

For the examples shown in Figs. 2 and 3 the performance of OMC stays constant with respect to increasing variable domains. This is because of the fact 
that, for these examples, the size of the fixpoint iterates and the number of fixpoint iterations stay constant with respect to increasing variable domains for the constraint-based model checker OMC. Note that, changing the maximum value that an integer variable can take from one integer constant to another integer constant does not increase the size of the constraint representation. Also, for the examples shown in Figures 2 and 3, the model checking procedure converges in a constant number of fixpoint iterations which is independent of the size of the domains of the variables. However, this may not always be the case. For example, for properties BOUNDED-BUFFER-3 and CIRCULAR-QUEUE-1 (Table 1) the number of fixpoint iterations depends on the size of the domain of the parameterized constant size.

Figure 2 shows the performances of SMV and OMC on verification of both two and three process implementations of the bakery algorithm with respect to the property BAKERY shown in Table 1. The performance of both SMV and $\mathrm{OMC}$ deteriorate significantly if the number of processes is increased. However, the cost of constraint-based model checking seems to increase more significantly compared to BDD-based model checking.

Based on Figs. 2 and 3 OMC outperforms SMV without interleaved variable ordering if the integer variables require more than 6 boolean variables to encode. If interleaved variable ordering $\left[\mathrm{CAB}^{+} 98\right]$ is used, for BAKERY with two processes and BARBER, the execution time of OMC is better than SMV if 18 and 14 boolean variables are used, respectively. The memory usage of OMC is always better than SMV in these cases. For the BAKERY with three processes SMV with interleaved variable ordering always outperforms OMC both in execution time and memory usage. For READERS-WRITERS, BOUNDED-BUFFER and CIRCULAR-QUEUE, OMC always outperforms SMV with interleaved variable ordering both in terms of execution time and memory usage.

Note that both bakery and barber algorithms given in Fig. 1 use variables with finite domains $\left(p c_{1}, p c_{2}, p c_{3}\right)$. Presence of such variables increases the cost of the constraint based representation since OMC partitions the state space to eliminate them. We believe that this is why the relative performance of OMC is not as good for these examples as it is for readers-writers, bounded-buffer and circular-queue. A composite approach which combines the BDD and constraintbased representations can be used in such cases [BGL98].

In Table 2 we show the performance of SMV (with interleaved variable ordering) and OMC for the problem instances given in Table 1 where each integer variable $i$ is restricted to the range $0 \leq i<1024$ (we also restricted the parameterized constant size to $0 \leq$ size $<16$ ). For most of these instances SMV and OMC have comparable performances. However for the BAKERY the increase in execution time and memory usage of $\mathrm{OMC}$ with respect to increasing number of processes is significantly higher compared to SMV. For 4 processes OMC did not converge in one hour (we indicate this with $\uparrow$ in Table 2 ).

Another shortcoming of OMC is demonstrated in the verification of properties BOUNDED-BUFFER-3 and CIRCULAR-QUEUE-1 shown in Table 1. None of the fixpoint computations for these properties converged (in an hour) when we 
Table 2. Experiments where each integer variable $i$ is restricted to $0 \leq i<1024$. In bounded-buffer and circular-queue instances the parameterized constant size is restricted to $0 \leq$ size $<16$ ( $\uparrow$ denotes that the fixpoint computations did not converge)

\begin{tabular}{|c|c|c|c|c|}
\hline \multirow[b]{2}{*}{ Problem Instance } & \multicolumn{2}{|c|}{\begin{tabular}{|c|} 
SMV (with Chan's \\
variable ordering)
\end{tabular}} & \multicolumn{2}{|c|}{$\mathrm{OMC}$} \\
\hline & \begin{tabular}{|c|} 
Execution \\
Time \\
(seconds)
\end{tabular} & \begin{tabular}{|c|} 
Memory \\
Usage \\
(Kbytes)
\end{tabular} & $\begin{array}{c}\text { Execution } \\
\text { Time } \\
\text { (seconds) }\end{array}$ & $\begin{array}{l}\text { Memory } \\
\text { Usage } \\
\text { (Kbytes) }\end{array}$ \\
\hline BAKERY (2 processes) & 0.12 & 1507 & 0.29 & 655 \\
\hline BAKERY (3 processes) & 0.82 & 2228 & 7.32 & 12165 \\
\hline BAKERY (4 processes) & 19.15 & 9110 & $\uparrow$ & $\uparrow$ \\
\hline BARBER & 0.40 & 2425 & 0.55 & 1458 \\
\hline BARBER-1 & 0.53 & 2490 & 15.37 & 23101 \\
\hline BARBER-2 & 0.35 & 2228 & 0.29 & 926 \\
\hline BARBER-3 & 0.35 & 2228 & 0.15 & 655 \\
\hline READERS-WRITERS & 0.03 & 1245 & 0.05 & 295 \\
\hline BOUNDED-BUFFER & 0.28 & 2163 & 0.08 & 238 \\
\hline BOUNDED-BUFFER-1 & 0.27 & 2228 & 0.05 & 188 \\
\hline BOUNDED-BUFFER-2 & 0.26 & 2163 & 0.04 & 147 \\
\hline BOUNDED-BUFFER-3 & 163.30 & 3080 & $\uparrow$ & $\uparrow$ \\
\hline CIRCULAR-QUEUE & 1.08 & 3408 & 0.10 & 377 \\
\hline CIRCULAR-QUEUE- 1 & 1228.45 & 6357 & $\uparrow$ & $\uparrow$ \\
\hline CIRCULAR-QUEUE-2 & 1.04 & 3342 & 0.07 & 328 \\
\hline
\end{tabular}

tried to verify them using OMC. This is the price we pay for using an expressive representation such as constraints which have higher worst case complexity than BDD manipulation. For the properties BOUNDED-BUFFER-3 and CIRCULARQUEUE-1, the number of fixpoint iterations depend on the size of the domain of the parameterized constant size. For these properties OMC does not converge even for the small domain $0 \leq$ size $<16$. Note that, for these cases BDD based model checking is not very efficient either (even with interleaved variable ordering). We think that for such cases using conservative approximation techniques would be helpful [BGP99].

\section{Conclusions}

The experimental results we obtained in this work suggests that constraintbased model checking can be more efficient than BDD-based model checking for verification of asynchronous concurrent systems with finite but large integer domains. This supports our view that constraint-based model checking is not limited to infinite-state systems but can also be useful for verification of systems with large integer domains. 

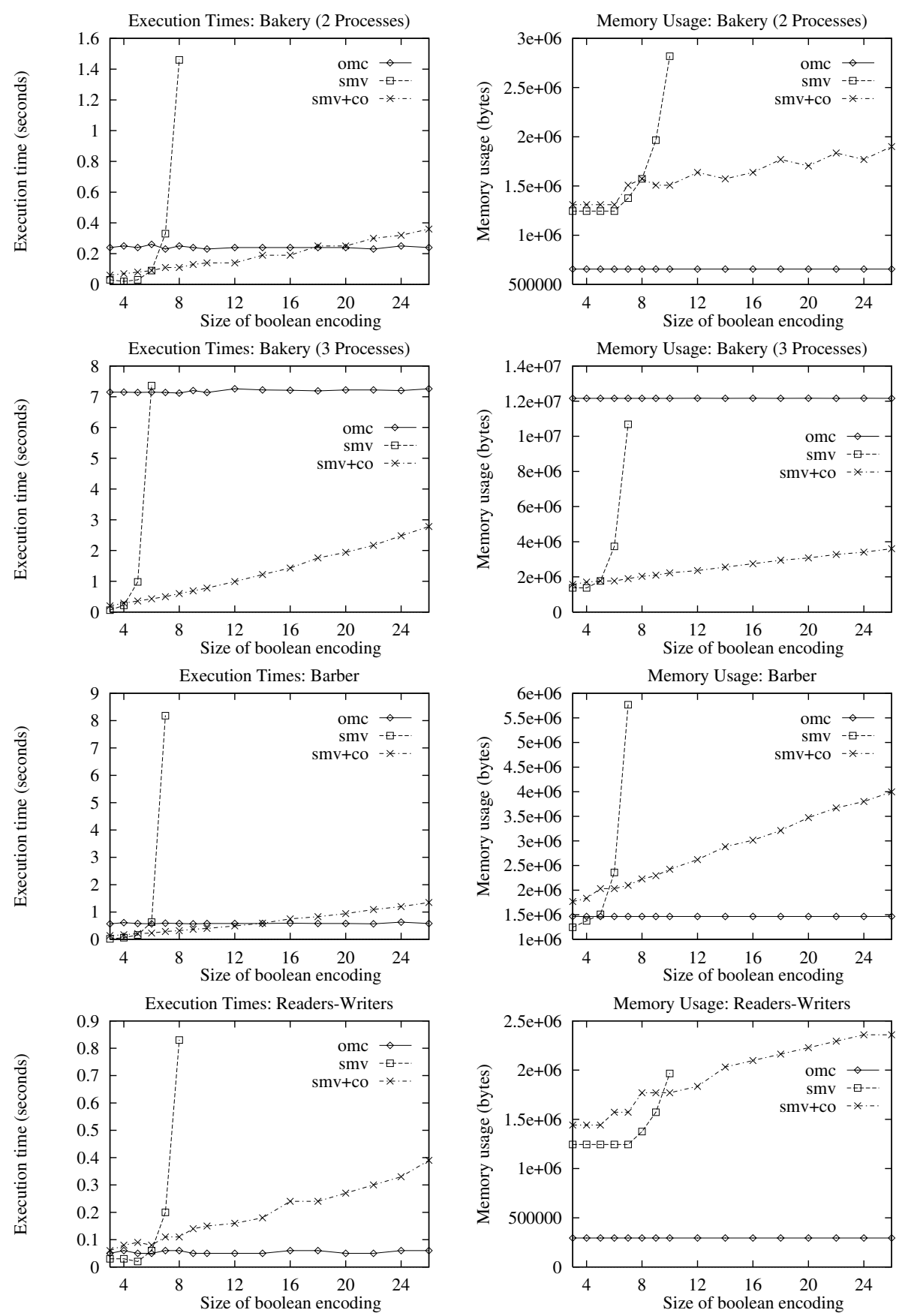

Fig. 2. Execution times and memory usage for OMC, SMV, and SMV with Chan's variable ordering $(s m v+c o)$ in verification of BAKERY, BARBER and READERS-WRITERS 

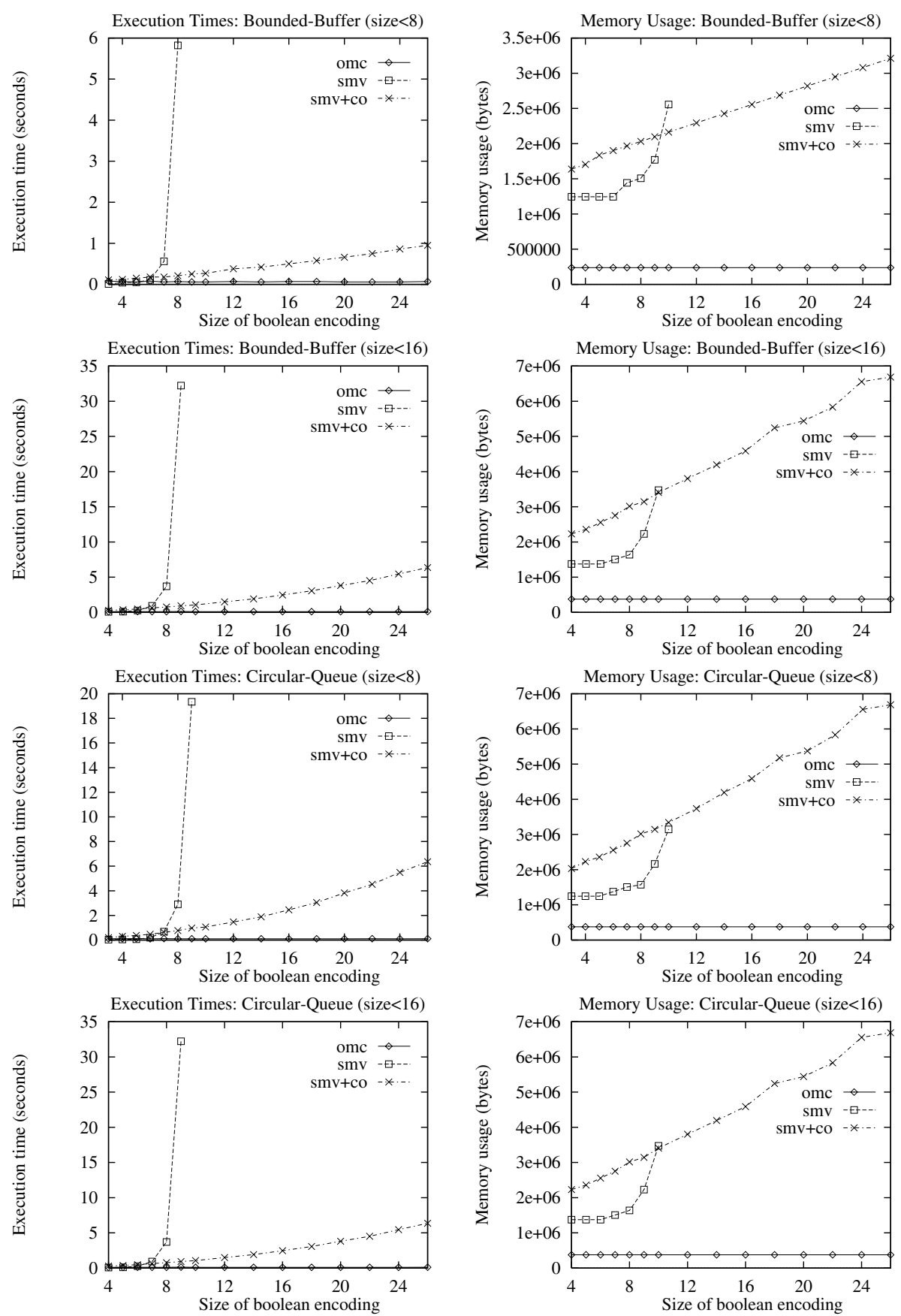

Fig. 3. Execution times and memory usage for OMC, SMV, and SMV with Chan's variable ordering $(\mathrm{smv}+\mathrm{co})$ in verification of BOUNDED-BUFFER and CIRCULAR-QUEUE 
In the future we would like to compare the performance of constraint-based model checking with the performance of word-level model checking [CZ95]. We are also planning to investigate the performance of our composite model checking approach [BGL98] with respect to BDD-based representations.

We would also like to investigate the complexity analysis of both BDD and constraint-based model checking for the type of systems analyzed in this paper.

\section{Acknowledgments}

This work was significantly improved by William Chan who provided insightful comments for an earlier draft of this paper and allowed access to his macros for interleaved variable order generation for SMV. Tragically, few weeks after our correspondence William Chan was killed in an accident. His research contributions and friendship will be greatly missed by me and our research community.

\section{References}

AHH96. R. Alur, T. A. Henzinger, and P. Ho. Automatic symbolic verification of embedded systems. IEEE Transactions on Software Engineering, 22(3):181201, March 1996. 442

And91. G. R. Andrews. Concurrent Programming: Principles and Practice. The Benjamin/Cummings Publishing Company, Redwood City, California, 1991. 446

BCL91. J. R. Burch, E. M. Clarke, and D. E. Long. Symbolic model checking with partitioned transition relations. In Proceedings of the International Conference on Very Large Scale Integration, August 1991. 445

$\mathrm{BCM}^{+}$90. J. R. Burch, E. M. Clarke, K. L. McMillan, D. L. Dill, and L. H. Hwang. Symbolic model checking: $10^{20}$ states and beyond. In Proceedings of the 5th Annual IEEE Symposium on Logic in Computer Science, pages 428-439, January 1990. 441, 444, 446

BGL98. T. Bultan, R. Gerber, and C. League. Verifying systems with integer constraints and boolean predicates: A composite approach. In Proceedings of the 1998 ACM SIGSOFT International Symposium on Software Testing and Analysis, pages 113-123, March 1998. 450, 454

BGP97. T. Bultan, R. Gerber, and W. Pugh. Symbolic model checking of infinite state systems using Presburger arithmetic. In O. Grumberg, editor, Proceedings of the 9th International Conference on Computer Aided Verification, volume 1254 of Lecture Notes in Computer Science, pages 400-411. Springer, June 1997. 442, 445, 446

BGP99. T. Bultan, R. Gerber, and W. Pugh. Model-checking concurrent systems with unbounded integer variables: Symbolic representations, approximations, and experimental results. ACM Transactions on Programming Languages and Systems, 21(4):747-789, July 1999. 442, 445, 446, 449, 451

BKR96. M. Biehl, N. Klarlund, and T. Rauhe. Mona: Decidable arithmetic in practice. In Proceedings of Formal Techniques in Real-Time and Fault-Tolerant Systems, 4th International Symposium, volume 1135 of Lecture Notes in Computer Science. Springer, 1996. 443 
Bry86. R. E. Bryant. Graph-based algorithms for boolean function manipulation. IEEE Transactions on Computers, 35(8):677-691, 1986. 441, 443, 446, 449

$\mathrm{CAB}^{+}$98. W. Chan, R. J. Anderson, P. Beame, S. Burns, F. Modugno, D. Notkin, and J. D. Reese. Model checking large software specifications. IEEE Transactions on Software Engineering, 24(7):498-520, July 1998. 441, 443, 449, 450

CABN97. W. Chan, R. J. Anderson, P. Beame, and D. Notkin. Combining constraint solving and symbolic model checking for a class of systems with non-linear constraints. In O. Grumberg, editor, Proceedings of the 9th International Conference on Computer Aided Verification, volume 1254 of Lecture Notes in Computer Science, pages 316-327. Springer, June 1997. 443

CES86. E. M. Clarke, E. A. Emerson, and A. P. Sistla. Automatic verification of finite-state concurrent systems using temporal logic specifications. ACM Transactions on Programming Languages and Systems, 8(2):244-263, April 1986. 444

CGL92. E. M. Clarke, O. Grumberg, and D. E. Long. Model checking and abstraction. In Proceedings of the 18th Annual ACM Symposium on Principles of Programming Languages, pages 343-354, 1992. 444

CZ95. E. Clarke and X. Zhao. Word level symbolic model checking: A new approach for verifying arithmetic circuits. Technical Report CMU-CS-95-161, School of Computer Science, Carnegie Mellon University, May 1995. 454

DP99. G. Delzanno and A. Podelski. Model checking in CLP. In Rance Cleaveland, editor, Proceedings of the 5th International Conference on Tools and Algorithms for the Construction and Analysis of Systems, volume 1579 of Lecture Notes in Computer Science, pages 223-239. Springer, March 1999. 442

$\mathrm{HKL}^{+}$98. C. L. Heitmeyer, J. Kirby, B. Labaw, M. Archer, and R. Bharadwaj. Using abstraction and model checking to detect safety violations in requirements specifications. IEEE Transactions on Software Engineering, 24(11):927948, November 1998. 444

$\mathrm{KMM}^{+}$97. Y. Kesten, O. Maler, M. Marcus, A. Pnueli, and E. Shahar. Symbolic model checking with rich assertional languages. In O. Grumberg, editor, Proceedings of the 9th International Conference on Computer Aided Verification, volume 1254 of Lecture Notes in Computer Science, pages 400-411. Springer, June 1997. 446

$\mathrm{KMP}^{+}$95. W. Kelly, V. Maslov, W. Pugh, E. Rosser, T. Shpeisman, and D. Wonnacott. The Omega library interface guide. Technical Report CS-TR-3445, Department of Computer Science, University of Maryland, College Park, March 1995. 442, 446

KSA98. J. H. Kukula, T. R. Shiple, and A. Aziz. Implicit state enumeration for FSMs with datapaths. In Proceedings of Formal Methods in ComputerAided Design, 1998. 443

McM93. K. L. McMillan. Symbolic model checking. Kluwer Academic Publishers, Massachusetts, 1993. 441, 442, 443, 446

SKR98. T. R. Shiple, J. H. Kukula, and R. K. Ranjan. A comparison of Presburger engines for EFSM reachability. In Proceedings of the 10th International Conference on Computer-Aided Verification, 1998. 443

WB95. P. Wolper and B. Boigelot. An automata-theoretic approach to Presburger arithmetic constraints. In Proceedings of the Static Analysis Symposium, September 1995. 443 
YMW97. J. Yang, A. K. Mok, and F. Wang. Symbolic model checking for eventdriven real-time systems. ACM Transactions on Programming Languages and Systems, 19(2):386-412, March 1997. 443, 449 